\title{
RFIA: A Novel RF-band Interference Attenuation Method in Passive Radar
}

\author{
Zeinab Shamaee, Mohsen Mivehchy \\ Department of Engineering, University of Isfahan, Isfahan, Iran \\ Email address: \\ z.shamaee@eng.ui.ac.ir (Z. Shamaee), mivehchy@eng.ui.ac.ir (M. Mivehchy)
}

\section{To cite this article:}

Zeinab Shamaee, Mohsen Mivehchy. RFIA: A Novel RF-band Interference Attenuation Method in Passive Radar. Journal of Electrical and Electronic Engineering. Vol. 4, No. 3, 2016, pp. 57-62. doi: 10.11648/j.jeee.20160403.13

Received: May 2, 2016; Accepted: May 11, 2016; Published: May 25, 2016

\begin{abstract}
Passive radars get benefit from transmitter signals in the environment for target detection. One of the most important challenges in these radars is multipath and direct path interferences that enter the target and reference antennas. This article expressed the types of weakening, and multi-step structure to mitigate the mentioned signals in passive radars. In the RF-band Interference Attenuation (RFIA) Method, at the first step the strongest interference component is mitigated by controlling the phase and amplitude. In next steps, other interference components are similarly nulled. This structure leads to separate mitigation for each interference components. Simulation results show the success of RFIA method to reduce dynamic range of analog-to-digital converter and, accordingly, the number of required bits for this converter.
\end{abstract}

Keywords: Passive Radar, Nulling, Interference Signal, Dynamic Range

\section{Introduction}

Passive radar has received much attention in the past decade. Conventional Radars consist of two parts including transmitter and receiver, that they are vulnerable, due to radiation [1-3]. Passive radar has one or more receivers and no dedicated transmitter and get advantage the exist signals in the environment for detecting targets. Since passive radars do not have access to modulating signal, they classified as bistatic radars. Fig. 1 shows the state of a passive bistatic radar [4].

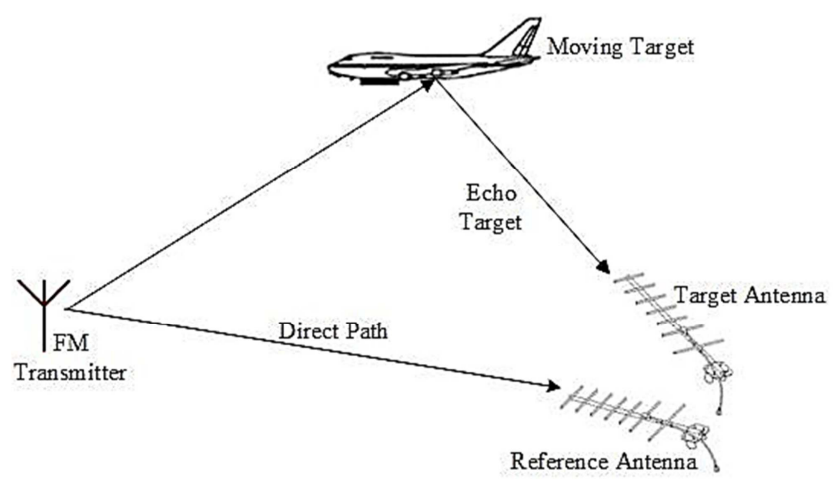

Figure 1. State of passive bistatic radar.
This radar composed of reference and target antenna. The reference antenna directly receives the signal from the intended transmitter as reference signal, and the task of target antenna is to receive the return signal from the target.

One of the most important issues in passive radar is direct path and multipath interferences (DPI \& MPI) that enter mentioned antenna besides the target and the reference signals. These strong interferences cause to enlarge the required dynamic range for analog to digital converter (A/D), and for weak signal it can leads to missing of the target. Also, should be careful to saturation of amplifiers in signal paths [5].

For reduction of dynamic range of entered signals to A/D converter, this article tried to offer RFICPR method for effective mitigation of direct path and multipath interference. In the RFIA method, a structure has been used which, first, reference signal is recovered without interferences and second, the strongest component of the interference signal as DPI is canceled from the target antenna signal. Then, in the further steps, the other interference components are suppressed step by step. Interference signals step mitigation characteristics include:

- Separate mitigating for each of interference component in each step and its implication for both the target and the reference signal

- More control capabilities on the appropriate amplitude 
and phase for a specific component in each step in order to further mitigation of that component.

In section 2, related literature in interference signal mitigation field are reviewed. Then, in section 3, mitigation basic concepts and the RFIA method are discussed. Besides, section 4 deals with evaluation and stimulation of the method, and section 5 presents the conclusion.

\section{Literature Work}

A set of available methods are trying to solve the problem in software method for reducing signal interference. In these methods, cancellation has been done in digital domain by various adaptive methods to filter out interference [6- 9]. Software methods placed after the A/D converter, and don't have effects on the required dynamic range for input of converters [10-12].

Use of physical shielding is one of the first hardware methods. Manastash Ridge Radar is the most important example for this method, in which the receiver placed on the other side of a large mountain [4].

Another hardware methods is adaptive beam forming, in which steering nulls towards the illuminator can lead to canceling the interference; although, the use of array antenna, has more nulls and finally has further canceling [13]. The main problem in these methods is increasing complexity of hardware systems which can be seen in Fig. 2.

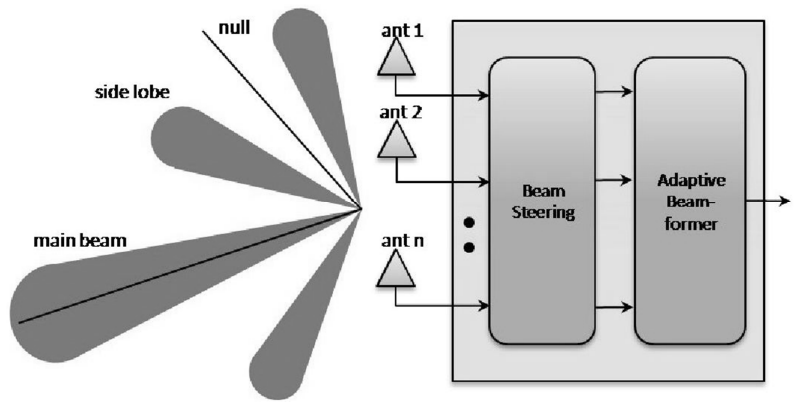

Figure 2. Adaptive beam forming scheme [14].

Another method is the use of high gain antenna that has a deeper null and more interference cancelling (Fig. 3), but it is impossible to build an antenna with high gain in low frequencies [15].

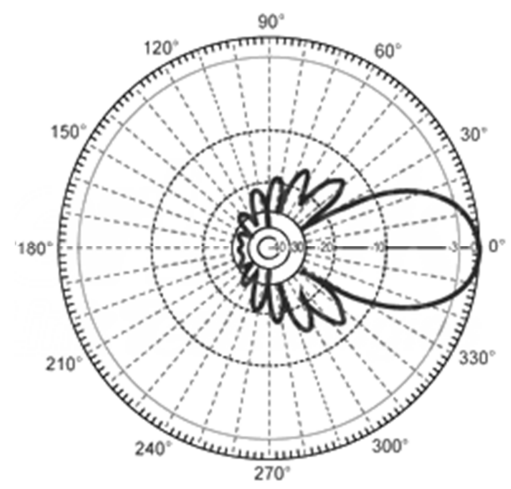

Figure 3. A typical pattern of high gain antenna [16]
In particular statuses, an antenna with polarization perpendicular to the polarization signal transmitter (Fig. 4) can be used to mitigate DPI for some extent. But this method is not that effective and can't have much impact on clutters [17].

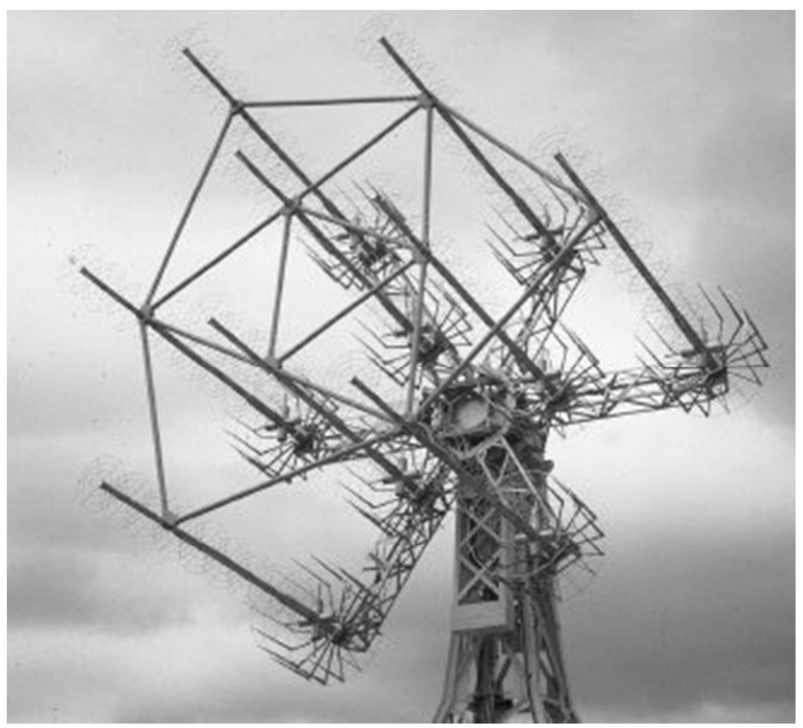

Figure 4. One of the Disk on rod antennas.

Another hardware that can be seen in Fig. 5 is based on the difference between the reference signals from target in analog domain [18]. The reference signal and the received direct path interference are coherent, but time of arrival (TOA) and amplitude of two signals are different. Since the base of interference cancellation in this method is subtraction between the target channel and the reference channel, therefore, the reference signal and DPI in target channel must be matched in amplitude and TOA.

In [17] with using this hardware method which is placed before $\mathrm{A} / \mathrm{D}$ converter, $35 \mathrm{~dB}$ mitigation is achieved, which is not enough. In order to improve the amount of mitigation in the proposed method, by staging cancelation of different components, totally, more mitigation can be achieved.

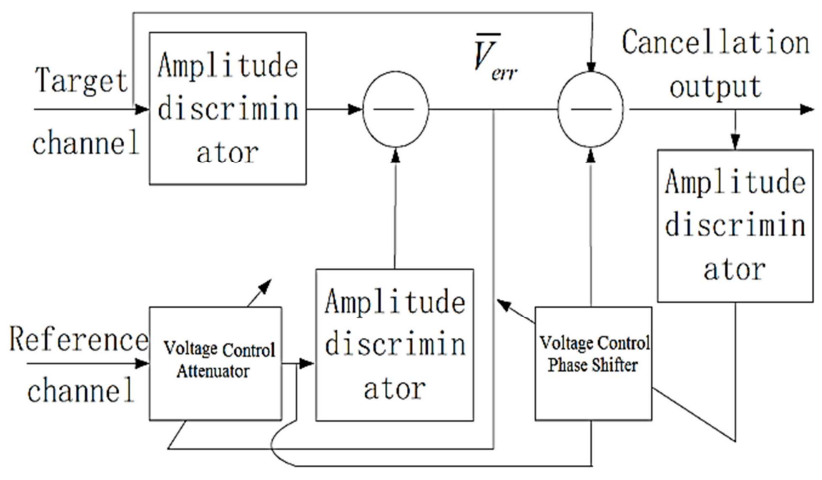

Figure 5. DPI cancellation with conventional analog method [17].

According to previous studies, multi-step nulling and in accordance with a specific component seems to be effective. Therefore, this paper tried to provide a method based on this structure. Details of the proposed method will be described in the next section. 


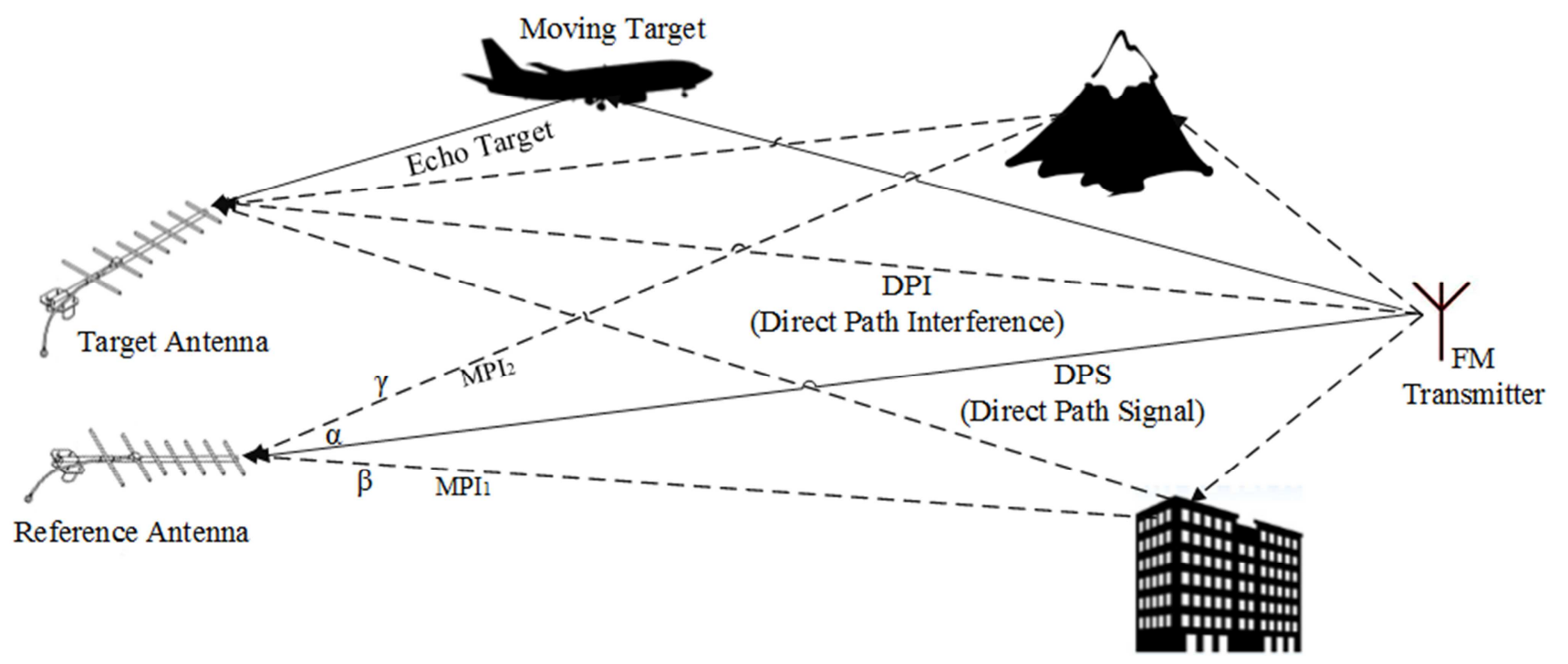

Figure 6. Applicable signals state and interference signals in passive radar.

\section{RFIA Method}

In passive radar, as seen in Fig. 6, the reference signal that carry out the process of passive radar, other interference signal unintentionally and simultaneously are received. This problem also occurs in the target antenna, where in addition to the target echo, strong interference such as DPI and returning interference are received from fixed objects placed in different distance from transmitter and receiver and have different amplitude and delays [20, 21].

All these are in situation that the radar cross section is much smaller than the DPI and multipath interference, For example, mountain ranges that make the interferences have a length of several kilometers and are distributed near the antennas. The difference between target signal power and interference signals sometimes reaches to $100 \mathrm{~dB}$, have other several issues that in first step needs using the A/D converter with many bits. In a typical example to cover the dynamic range of received signals about $100 \mathrm{~dB}$ need A/D converter with at least 17 bits, and this estimate is in the absence of noise, because in the noise presence, the number of required bits increases [22].

In proposed method for better attenuation of a desired component in each step, it is necessary to dominate the desired component in reference antenna signal with an appropriate method. So a new reference signal is produced and gathered by received signal from the target antenna. The result of this addition is attenuation of direct path components in the received signal from the target antenna, this process is called nulling. But, if over time and under environmental conditions, the received signal changed a little, the nulling is not done properly, and even can worsen the result. But in case of proper functioning after the first step of nulling, reduction interference about 20 to $30 \mathrm{~dB}$ is expected. And further reduction in one step is impossible due to existing more interference components with different delay, amplitude and phase.
In fact the nulling process performs in several steps on received signals; and one of the interference components in each step of attenuation is considered. In order to have a proper process in the second step, it is needed to mitigate DPI simultaneously in the path of received signal from the reference antenna.

To simplify analysis, information signal $\mathrm{x}(\mathrm{t})$ in $(1)$, is supposed as a sine wave with $f_{m}$ frequency $\left(\omega_{m}=2 \pi f_{m}\right)$. Equation (2) expresses carrier signal that frequency modulated by $x(t)$ and $f_{\Delta}$ is maximum instantaneous frequency deviation around central frequency $\left(\omega_{c}=2 \pi f_{c}\right)$.

$$
\begin{aligned}
x(t) & =\sin \omega_{m} t \\
z(t) & =\sin \left[\omega_{c}+2 \pi f_{\Delta} \cos \left(\omega_{m} t\right)\right] t
\end{aligned}
$$

Signal $z(t)$ with amplitude $\alpha$ and delay $t_{d 1}$ as DPS accompany with two interference signal with $\beta$ and $\gamma$ values as amplitude and delays $t_{d 2}$ and $t_{d 3}$, namely $R(t)$ in (3) where $\alpha$ is greater than $\beta$ and $\gamma$ and $t_{d 1}$ is less than $t_{d 2}$ and $t_{d 3}$.

$$
\begin{aligned}
& R(t)=\alpha \sin \left[\left[\omega_{c}+2 \pi f_{\Delta} \cos \omega_{m}\left(t-t_{d 1}\right)\right]\left(t-t_{d 1}\right)\right] \\
& \quad+\beta \sin \left[\left[\omega_{c}+2 \pi f_{\Delta} \cos \omega_{m}\left(t-t_{d 2}\right)\right]\left(t-t_{d 2}\right)\right] \\
& \quad+\gamma \sin \left[\left[\omega_{c}+2 \pi f_{\Delta} \cos \omega_{m}\left(t-t_{d 3}\right)\right]\left(t-t_{d 3}\right)\right]
\end{aligned}
$$

In (4), the signal $R(t)$ with $\operatorname{nr}(t)$ noise enters to the reference antenna $(\operatorname{Ref}(\mathrm{t}))$.

$$
\operatorname{Ref}(t)=R(t)+n_{r}
$$

On the other hand, transmitter signal goes to target antenna with as amplitude after hitting the moving target produces Doppler frequency fd. Finally information signal enters to target antenna with a coefficient $(\mathrm{k})$ of the reference signal and ns ( $\mathrm{t}$ ) noise, as $\mathrm{S}(\mathrm{t})$, that is expressed by (5).

$$
S(t)=k R(t)+T(t)+n_{s}(t)
$$



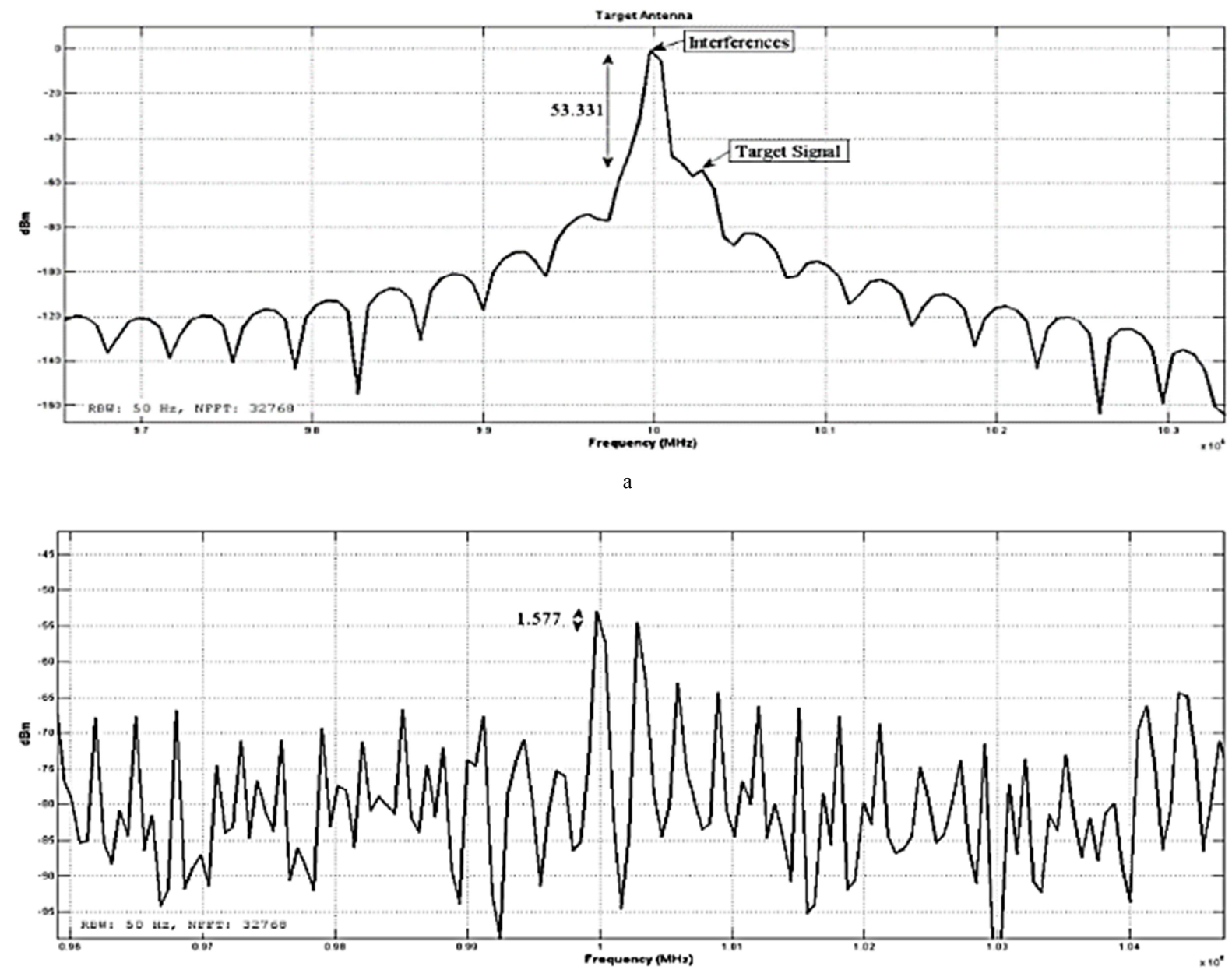

b

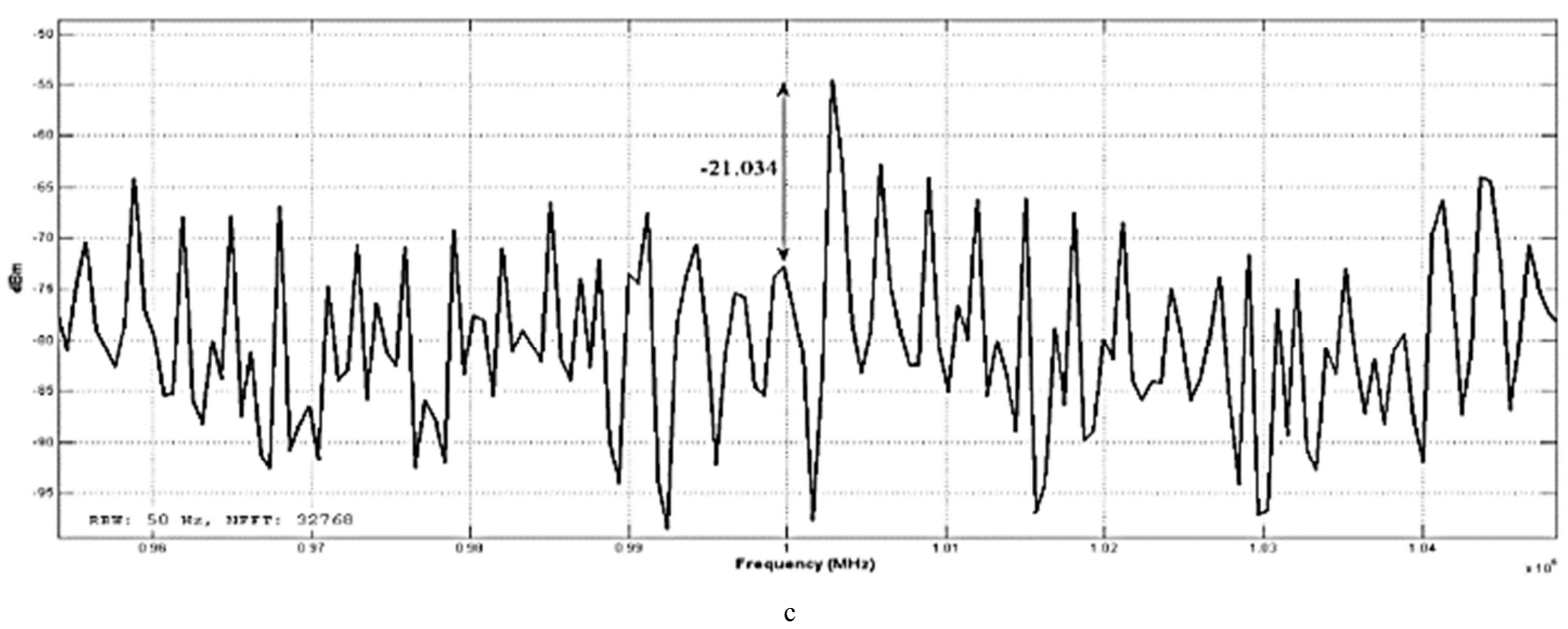

Figure 7. Frequency spectrum of target antenna signal. a. Before nulling. b. After first step attenuation. $c$. after second step attenuation.

\section{Evaluation}

Simulation of the RFIA method and also producing required signal for evaluation are done in MATLAB software. The RFIA method is implemented in two steps. In this simulation, the intended transmitter is considered from the FM type with carrier frequency of $100 \mathrm{MHz}$ and the speed target is about 0.9
Mach and Doppler frequency $\left(\mathrm{f}_{\mathrm{d}}\right)$ is about $300 \mathrm{~Hz}$. Evaluation Target signal frequency spectrum with the interference components entered target antenna before nulling operations; is shown in Fig. 7.a. The target signal are placed at a frequency of $300 \mathrm{kHz}$ from the carrier.

Conventional analog method that described in literature work, is based on subtracting between reference and target 
antenna signal with appropriate phase and amplitude. With this method, $54 \mathrm{~dB}$ attenuation achieved.

Spectrum of target signal after maximum attenuation by first step of RFIA method is shown in Fig. 7.b. Prior to nulling, the target component power difference with the strongest component of interference components, is about $53 \mathrm{~dB}$. But after nulling this difference reached to 1.577. This amount of mitigation, is almost the highest weakening in one step. Because of presence of the next interference component that requires a separate setting of amplitude and phase shift, mitigating in this step is limited up to this rate. For further mitigations, the next steps should be passed. Frequency spectrum of target antenna signal after second step attenuation is depicted in Fig. 7.c.

The spectrum is related to the optimum gain and phase, so the amount of overall attenuation is about $73 \mathrm{~dB}$. By comparing the conventional method attenuation $(54 \mathrm{~dB})$ and the attenuation of RFIA method $(73 \mathrm{~dB})$ an improvement about $20 \mathrm{~dB}$ is obtained as shown in Fig. 8 .

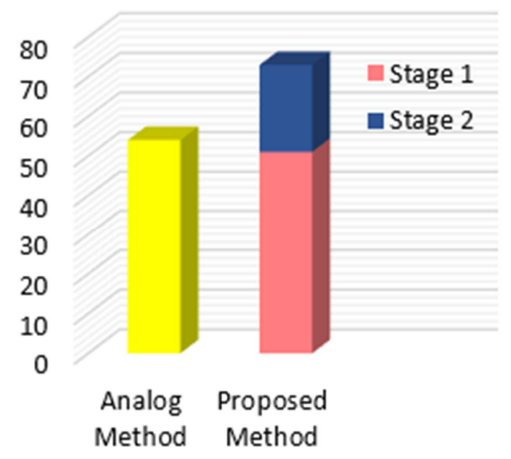

Figure 8. Comparison between conventional and RFIA method.

In RFIA method, About $51 \mathrm{~dB}$ Attenuation in one step obtained. However in the conventional analog method, the maximum attenuation was about $54 \mathrm{~dB}$. The reason behind this discrepancy is that in the multi-step method, due to dominating process, in each step the strongest component itself attenuates with the highest possible amount and the power of other components does not change. But in the conventional method, with attenuating first component, the other components also slightly attenuates. But, finally, in the two step method after accomplishment of the second step, other interference component are also attenuate and generally better outcome is obtained compared to its conventional analog method.

Totally by RFIA method, the dynamic range from the target antenna dropped at least about $20 \mathrm{~dB}$ more than the conventional analog method, which means approximately 3 bits fewer for $\mathrm{A} / \mathrm{D}$ converter input.

The distance between receiver antennas can play a major rule in the maximum limit for interference attenuation. So, the effect of delay between signals which arrives two antennas is not negligible and must be considered. The result of conventional analog method with effect of distances between receiver antennas show the maximum attenuation $39 \mathrm{~dB}$. With completion of the proposed method and after second step, the amount of the overall attenuation is $50 \mathrm{~dB}$ which is about 11 $\mathrm{dB}$ higher than the conventional analog method. These results show the proposed method is successful in more attenuation of interferences.

\section{Conclusion}

This paper presented a multi-step structural method to weaken the direct path and multipath interference components in passive radars. In the first step of RFIA method, DPI (the strongest interference component) and in further step, the other components are attenuated. To enter the next step, mitigation first of the interference component is applied in the reference antenna in addition to the target antenna. Indeed, for each step a new reference signal generated.

In comparison with earlier works, the mitigation ability of this method is about $11 \mathrm{~dB}$ more; and at the same time it should be taken in to account that, this interference components mitigation is done before $\mathrm{A} / \mathrm{D}$ converter. For more cancelation of the remained interference components, still software methods can be used.

The success of the RFIA method depends on this proper amplitude and phase shift proportionate for any components. Thus offering an alternative for its automatic determining for various issues is needed.

\section{References}

[1] H. Kuschel and D. O'Hagan, "Passive radar from history to future," Radar Symp. (IRS), 2010 11th Int., 2010.

[2] P. Howland, "Editorial: Passive radar systems," IEE Proc. Radar, Sonar Navig., vol. 152, no. 3, p. 105, 2005.

[3] H. Kuschel and F. Fhr, "T10 - Passive bistatic radar, theory Systems - Measurements," in 2014 IEEE Radar Conference, 2014, pp. 37-37.

[4] H. D. G. and C. J. B. Paul E. Howland, Bistatic Radar: Emerging Technology. Chichester, UK: John Wiley \& Sons, Ltd, 2008, pp. 247-313.

[5] J. Brown, "FM airborne passive radar-Phd Thesis," UCL (University College London), 2013.

[6] J. Palmer and S. Searle, "Evaluation of adaptive filter algorithms for clutter cancellation in passive bistatic radar," Radar Conf. (RADAR), 2012 IEEE, pp. 0493-0498, May 2012.

[7] F. Colone, R. Cardinali, and P. Lombardo, "Cancellation of Clutter and Multipath in Passive Radar using a Sequential Approach," in 2006 IEEE Conference on Radar, 2006, pp. 393-399.

[8] X. Guan, D.-H. Hu, L.-H. Zhong, and C.-B. Ding, "Strong Echo Cancellation Based on Adaptive Block Notch Filter in Passive Radar," IEEE Geosci. Remote Sens. Lett., vol. 12, no. 2, pp. 339-343, Feb. 2015.

[9] B. W.; C. L.; C. C.; W. C. Feng, "An effective CLEAN algorithm for interference cancellation and weak target detection in passive radar," in Synthetic Aperture Radar (APSAR), 2013 Asia-Pacific Conference on, 2013, pp. 160-163. 
[10] Z. Shamaee, M. Mivehchy, and M. F. Sabahi, "A Chi-square-based induction method for interfernce signals attenuation in passive radar," in The Third Iranian Conference of Engineering Electromagnetics, 2015.

[11] P. E. Howland, D. Maksimiuk, and G. Reitsma, "FM radio based bistatic radar," IEE Proceedings - Radar, Sonar and Navigation, vol. 152, no. 3. p. 107, 2005.

[12] B. Farhang-Boroujeny, Adaptive Filters: Theory and Applications, Second. Chichester, UK: John Wiley \& Sons, Ltd, 2013.

[13] G. Fabrizio, F. Colone, P. Lombardo, and a. Farina, "Adaptive beamforming for high-frequency over-the-horizon passive radar," IET Radar, Sonar Navig., vol. 3, no. 4, p. 384, 2009.

[14] http://www.telecomfamily.com. [Accessed: 10- May- 2016].

[15] C. Coleman, "Mitigating the Effect of Direct Signal Interference in Passive Bistatic Radar," 2009, no. red X, pp. 1-4.

[16] http://www.l-com.com. [Accessed: 10- May- 2016].
[17] R. Saini, M. Cherniakov, and V. Lenive, "Direct path interference suppression in bistatic system: DTV based radar," 2003 Proc. Int. Conf. Radar (IEEE Cat. No. 03EX695), 2003.

[18] C. L. Zoeller, M. C. Budge, and M. J. Moody, "Passive coherent location radar demonstration," in Proceedings of the Thirty-Fourth Southeastern Symposium on System Theory (Cat. No. 02EX540), 2002, pp. 358-362.

[19] H. Wan, S. Li, and Z. Wang, "Direct Path Interference Cancellation in FM Radio-Based Passive Radar," in 2006 8th international Conference on Signal Processing, 2006, vol. 3, pp. 4-7.

[20] H. Griffiths and C. Baker, "The Signal and Interference Environment in Passive Bistatic Radar," in 2007 Information, Decision and Control, 2007, pp. 1-10.

[21] H. Griffiths, "Passive Bistatic Radar and Waveform Diversity," vol. 119, no. c, 2009.

[22] M. Skolnik, Radar Handbook, 3rd ed. McGraw-Hill Professional; 3rd edition, 2008. 